\title{
181. Zum Wert palliativchirurgischer Eingriffe bei Mammacarcinom- metastasen im Bereiche des Skelets und des Zentralnervensystems
}

\author{
R. Margreiter, W. Russe, J. Fischer und J. Wiegele
}

I. Universitätsklinik für Chirurgie, Universitätsklinik für Orthopädie, Universitätsklinik für Neurochirurgie, Anichstraße 35, A-6020 Innsbruck

\section{Palliative Surgical Treatment of Bone and CNS Metastases from Breast Cancer}

Summary. From 1975 to 1981, 27 endoprostheses (hip 19, spine 3, humerus 3, entire femur 1, partial pelvic replacement 1) were implanted in breast cancer patients for osseous metastases; 16 oesteosyntheses (femur 11, spine 4, tibia 1) and 21 laminectomies were carried out; 11 solitary brain metastases were excised. The rehabilitation rate achieved was $95 \%$ after bone surgery and $100 \%$ after laminectomy. The indication for these procedure is an absolute. On the other hand, excision of brain metastases makes the patient symptom-free for a short period of time but does not alter the bad prognosis: all of these patients died within 7 months.

Key words: Breast cancer - Bone metastases - CNS metastases - Surgical treatment.

Zusammenfassung. Von 1975-1981 wurden an unseren Kliniken bei Mammacarcinompatientinnen wegen Knochenmetastasen 27 Endoprothesen (Hüfte 19, Wirbelsäule (WS) 3, Oberarm 3, gesamter Femur 1, Beckenteilersatz 1) implantiert, 16 Verbundosteosynthesen (Oberschenkel 11, WS 4, Unterschenkel 1) und 21 Laminektomien durchgeführt sowie 11 solitäre Hirnmetastasen operativ entfernt. $95 \%$ betrug die Rehabilitationsquote nach Eingriffen am Knochen, $100 \%$ nach Laminektomie. Die Indikation für diese Eingriffe ist eine absolute. Hingegen scheint die alleinige Entfernung von solitären Hirnmetastasen eine nur kurzzeitige Besserung zu bringen aber nichts an der schlechten Prognose zu ändern: Alle diese Frauen sind innerhalb von 7 Monaten verstorben.

Schliisselwörter: Brustkrebs - Knochenmetastasen - ZNS-Metastasen - Chirurgische Behandlung. 